\title{
Emotional Priming Effects during Stroop Task Performance
}

\author{
Sarah J. Hart ${ }^{1,3}$, Steven R. Green ${ }^{4}$, Michael Casp ${ }^{3}$, and Aysenil Belger ${ }^{1,2,3}$ \\ 1 UNC Neurodevelopmental Disorders Research Center, University of North Carolina at Chapel Hill, \\ Chapel Hill, NC \\ 2 UNC Department of Psychiatry, University of North Carolina at Chapel Hill School of Medicine, \\ Chapel Hill, NC \\ ${ }^{3}$ Duke-UNC Brain Imaging and Analysis Center, Duke University Medical Center, Durham, NC \\ ${ }^{4}$ Center for Cognitive Neuroscience, Duke University, Durham, NC
}

\section{Abstract}

The ability to make decisions within an emotional context requires a balance between two functionally integrated neural systems that primarily support executive control and affective processing. Several studies have demonstrated effects of emotional interference presented during an ongoing cognitive task, but it is unclear how activating the emotional circuitry prior to a cognitive task may enhance or disrupt the executive system. In this study we used fMRI to examine the effects of emotional priming on executive processing during a number Stroop task. Our results indicated that during trials with less executive requirements, there was a greater aversive emotional attenuation effect in a network of regions including the ventrolateral prefrontal cortex (vlPFC), insula and cingulate gyrus. This attenuation effect was counteracted during trials with increased executive demand, suggesting that while pre-activation of the emotional system may lead to an automatic attenuation of activity in multiple regions, requirements for executive function may override the aversive emotional attenuation effect. Furthermore, this override effect was found to be associated with faster reaction times during executive processing. These findings demonstrate that activity in the vlPFC, cingulate and insula is dynamically adjusted in order to optimize performance, and illustrate the importance of the timing of each system's engagement in determining how competing cognitive and emotional information is processed.

\section{Introduction}

The relationship between cognition and emotion has been a topic of recent interest for studies of both normal brain function and psychiatric diseases. The ability to make decisions under stressful or emotional contexts requires a balance between cognitive control for task-relevant information and orienting to salient information in the environment. Under conditions where cognitive and emotional information compete with one another, the presence of emotionally salient information has been found to disrupt the ability to attend to task-relevant information (Arnsten \& Goldman-Rakic, 1998; Dolcos \& McCarthy, 2006). However, other studies have demonstrated that the presence of emotional stimuli can enhance cognitive processing (Gray, Braver, \& Raichle, 2002; Hartley \& Adams, 1974; Schupp, Stockburger, Codispoti et al.,

Correspondence: Sarah J. Hart, Ph.D. Department of Psychiatry, CB \# 7160, University of North Carolina at Chapel Hill, Chapel Hill, NC 27599, Tel: 919-843-3153, Fax: 919-966-8004, sjhart@email.unc.edu.

Publisher's Disclaimer: This is a PDF file of an unedited manuscript that has been accepted for publication. As a service to our customers we are providing this early version of the manuscript. The manuscript will undergo copyediting, typesetting, and review of the resulting proof before it is published in its final citable form. Please note that during the production process errors may be discovered which could affect the content, and all legal disclaimers that apply to the journal pertain. 
2007). The precise nature of the relationship between cognition and emotion remains to be clarified.

The interaction between these functions is thought to reflect a balance between two neural systems, which have been described as the ventral affective processing system (VAPS) and dorsal executive control system (DECS) (Dolcos \& McCarthy, 2006). While emotionally salient stimuli are processed in VAPS regions such as the amygdala, orbitofrontal cortex, and medial prefrontal cortex, executive function recruits DECS regions including the dorsolateral prefrontal cortex (dlPFC), posterior parietal cortex, and anterior cingulate. These systems are highly integrated with one another, as reflected by patterns of functional and structural connectivity between VAPS and DECS regions (Barbas, 1995; Pessoa, 2008; Young, Scannell, Burns, \& Blakemore, 1994).

Several lines of evidence support this integrative model positing an interdependence between executive functions and affective processes (Gray, Braver, \& Raichle, 2002; Pessoa, 2008). It has been shown, for example, that selective attention processes are enhanced in the presence of emotionally significant stimuli (Vuilleumier, Richardson, Armony, Driver, \& Dolan, 2004). Alternatively, processing of emotional information also depends upon engagement of executive resources. Several studies have demonstrated that processing of emotional stimuli can be modulated by using a cognitive reappraisal strategy that downplays their emotional impact (Beauregard, Levesque, \& Bourgouin, 2001; Ochsner \& Gross, 2005). Processing of emotional stimuli is also susceptible to interference by taxing cognitive processing resources (Blair et al., 2007; Mitchell et al., 2007; Schupp, Stockburger, Bublatzky et al., 2007).

While these studies have demonstrated that emotional and executive processes are highly interactive, the nature of these interactions and their influence on behavior are unclear. In particular, the manner in which these systems interact when each is engaged to a different degree, as well as how the timing of each system's engagement affects the other, remain to be fully clarified. Previous studies investigating these interactions have typically examined how emotional stimuli create interference when presented during ongoing cognitive processing (Dolcos \& McCarthy, 2006), or by examining the executive response while attending to items with emotional or neutral content (Williams, Mathews, \& MacLeod, 1996). However, it has not yet been clearly determined how engaging the emotional system prior to an executive task may affect its processing. As it has been demonstrated that mildly stressful stimuli can actually improve task performance (Hartley \& Adams, 1974), emotional stimuli that precede an executive task could potentially provide an attentional bias towards the subsequent stimuli. Alternatively, emotional stimuli can divert attentional resources away from the subsequent executive task, resulting in impaired performance and decreased activation in the prefrontal cortex (Arnsten \& Goldman-Rakic, 1998). The timing of the task may be a critical variable influencing whether emotional stimuli have a beneficial or deleterious effect on cognitive processing, as emotional interference has been shown to have differential effects on perceptual processing in studies that present emotional information prior (Most, Chun, Widders, \& Zald, 2005) or subsequent to the task (Arnsten \& Goldman-Rakic, 1998). Testing the effects of priming of the affective system prior to engaging in an executive task would therefore provide important information on how the temporal dynamics of these systems influences cognitive processing and performance.

We therefore sought to answer whether pre-activation of the VAPS would elicit differential DECS engagement during an executive task, whether this effect would vary according to degree of DECS engagement, and how these interactions would influence behavior. In this study, we used a number Stroop task to manipulate the degree of DECS engagement (Blair et al., 2007; Pansky \& Algom, 2002). In this task, participants are asked to report the number of items presented in an array of digits, which can either be congruent or incongruent with the digit 
printed in the array. Responding to an incongruent array requires engagement of executive function in order to suppress the automatic response of reading the printed digit. The Stroop arrays in the current study were preceded by a briefly presented prime stimulus with either negative emotional (aversive) or neutral content, to manipulate the engagement of the VAPS.

Given that previous studies have demonstrated a deleterious effect of emotional stimuli presented prior to a perceptual task (Mathewson, Arnell, \& Mansfield, 2008; Most, Chun, Widders, \& Zald, 2005), we predicted that aversive primes would impair rather than enhance executive performance. We predicted that during incongruent trials, reaction times would show greater slowing following aversive primes than neutral primes, while congruent trials would show less of an effect of prime type on reaction times. Furthermore, we expected that the aversive primes would lead to relative deactivation in prefrontal areas relative to neutral primes, and that this effect would differ between incongruent and congruent trials. Finally, given that previous studies have demonstrated that emotional disruption of dlPFC function is associated with poorer performance (Dolcos \& McCarthy, 2006), we predicted that slower reaction times would be associated with deactivation in prefrontal areas during aversive prime trials.

\section{Materials and Methods}

\section{Participants}

Fourteen healthy volunteers participated in the functional imaging task. All participants provided informed written consent as approved by the Duke University Medical Center Institutional Review Board, and in compliance with national legislation and the Declaration of Helsinki. Exclusion criteria included presence of a neurological, psychiatric, or substance abuse disorder, presence of metal in the body, and pregnancy. The sample of participants had a mean age of 25.3 years (range 18-36 years) and included 5 males and 9 females.

\section{Imaging Task}

During functional imaging, participants performed a number Stroop task preceded by aversive or neutral pictures. During the number Stroop task, participants were presented with an array of $1,2,3$, or 4 digits for $1 \mathrm{sec}$ and were asked to indicate by button press the number of items presented in the array. The number of items could be congruent (e.g., the digit 4 in an array of 4) or incongruent (e.g., the digit 4 in an array of 3 ) with the printed digits. Congruent and incongruent trials each made up approximately $17 \%$ of the total trials. The remaining trials consisted of arrays of star-shaped stimuli, in order to provide a control counting condition without the task-irrelevant digit information.

Immediately preceding each array, a prime stimulus was presented for $150 \mathrm{msec}$ with either aversive or neutral content. Ninety total images were selected from the International Affective Picture System database (Lang, Bradley, \& Cuthbert, 2005), which contains complex pictures with standardized ratings for arousal and valence, rated on a scale of 1-9 (with lower numbers indicating less arousal and more negative valence). The full list of images used is presented in an Appendix. The aversive stimuli from this database have been found to consistently activate the emotional circuitry (Dichter, Bellion, Casp, \& Belger, 2008; Lane et al., 1997; Paradiso et al., 1999). T-tests confirmed that the aversive and neutral pictures significantly differed according to arousal $(F(1,88)=251.52, p<.0001)$ and valence $(F(1,88)=230.34, p<0001)$. Aversive pictures had an average valence of $3.36(S D=.72)$ and arousal of $6.19(S D=.63)$. Neutral pictures had an average valence of $6.24(S D=1.03)$ and arousal of $3.67(S D=.84)$. Primes were presented on all trials, including randomly intermixed aversive and neutral trials each making up 50\% of the total. The intertrial intervals varied randomly between 1500, 2000, and $2500 \mathrm{msec}$. A schematic of the task design is shown in Figure 1. There were 6 total runs presented, each containing 103 total trials and lasting around 5.5 minutes. Because there were 
more total trials than unique prime stimuli, the aversive and neutral pictures were repeated several times throughout the experiment. All stimuli were displayed using CIGAL software (Voyvodic, 1999).

\section{Image Acquisition}

The fMRI images were acquired on a General Electric 4.0 Tesla MRI scanner using a functional inverse spiral acquisition sequence allowing for full-brain coverage (TR: $2000 \mathrm{msec}$; TE: 6 msec; FOV: 256; image matrix: $64 \times 64$; Flip angle 60; Voxel size: $4 \times 4 \times 4 \mathrm{~mm}$; 34 axial slices). Functional runs for the primary task consisted of 161 time points. Preceding the functional image acquisition, we acquired structural 3D high resolution T1 images using a spoiled gradient-recalled acquisition pulse sequence (TR: $12.2 \mathrm{msec}$; TE: $5.3 \mathrm{msec}$; FOV: 250; image matrix: $256 \times 256$; Flip angle 20; Voxel size: $.98 \times .98 \times 2 \mathrm{~mm}$; 68 axial slices).

\section{Analyses}

\section{Preprocessing}

Functional data analyses were carried out using FSL version 4.0 [Oxford Centre for Functional Magnetic Resonance Imaging of the Brain (FMRIB), Oxford University, U.K.] (Smith et al., 2004). Image preprocessing (first-level analysis) steps included using the Brain Extraction Tool (BET) to remove non-brain structures (Smith, 2002), motion correction (Jenkinson, Bannister, Brady, \& Smith, 2002), spatial filtering using a Gaussian kernel of full width half maximum $5 \mathrm{~mm}$, high-pass temporal filtering, and time-slice correction. The functional images were coregistered to the structural images in their native space, and the images were then normalized to the Montreal Neurologic Institute (MNI) standard brain. All registrations were carried out using FMRIB's Linear Image Registration Tool (FLIRT) (Jenkinson \& Smith, 2001). Prewhitening was carried out with FMRIB's Improved Linear Model (FILM) in order to estimate and account for each voxel's time series autocorrelation (Woolrich, Ripley, Brady, \& Smith, 2001).

\section{Voxel-based Analyses}

All trials where participants gave an incorrect response to the Stroop array were excluded from the analyses. For the stars condition, we included a randomly selected subset of the total trials in order to equate the number of trials being compared between the three Stroop conditions. The rationale for this approach was to avoid spurious findings based on different signal-tonoise ratios between the stars condition and other conditions. The same subset of stars trials was used for all participants, and the remaining stars trials were not included in the analyses.

All voxel-based analyses were carried out using the FMRI Expert Analysis Tool (FEAT) in FSL. Onset times of events were used to model regressors for each experimental condition, which were convolved with a gamma function to model the hemodynamic response. In the first-level analysis each run of the task was modeled separately, followed by the second-level analysis in which the average activation across runs for each subject was calculated with a fixed-effects analysis. At the second level, whole-brain images of the parameter estimates and variance were generated for each subject, representing the average percent signal change from baseline for each experimental condition (Aversive-Congruent, Aversive-Incongruent, Aversive-Stars, Neutral-Congruent, Neutral-Incongruent, Neutral-Stars). Contrast images were then generated to create $z$-statistic activation maps where the estimates for each condition were non-zero, to determine where each individual condition showed significant activation. Contrast maps were also generated to compare the following conditions: 1) Aversive versus Neutral, 2) Congruent versus Incongruent, 3) Neutral-Incongruent versus AversiveIncongruent, 4) Neutral-Congruent versus Aversive-Congruent, and 5) Aversive-Stars versus Neutral-Stars. At the third-level analysis, the average of these contrast maps across subjects 
was estimated using FMRIB's Local Analysis of Mixed Effects (FLAME) (Beckmann, Jenkinson, \& Smith, 2003; Woolrich, Behrens, Beckmann, Jenkinson, \& Smith, 2004). Grouplevel activation maps were thresholded using a $Z$-statistic to define contiguous clusters of activation. Significant clusters for main conditions and between-condition contrasts were corrected for multiple comparisons using a false discovery rate (FDR) threshold of $p<.05$ (Genovese, Lazar, \& Nichols, 2002). Additionally, contrasts comparing conditions were masked so that only voxels that showed positive activation were examined.

\section{Correlation Analyses}

In order to assess the relationship between behavior and brain activation, reaction time (RT) was demeaned (where the mean was subtracted from each value) and entered as a covariate in the model with FSL. Each participant's demeaned reaction time averaged across congruent and incongruent trials (during correct trials only) was included in the model to determine which regions' activity showed a significant positive or negative relationship with RT. These analyses produced whole-brain activation maps where significant positive and negative correlations were found between RT and mean z-scores for clusters of activation within task conditions of interest. We measured the relationship between each individual's average reaction time and activation during the contrast between aversive and neutral primes on incongruent and congruent trials. Significant clusters of activation were identified by correcting for multiple comparisons using an FDR-corrected threshold of $p<.05$. To display correlation data within specific regions, we extracted percent signal change within all voxels in anatomical regions defined by the Harvard-Oxford Cortical Atlas in FSL and plotted these values against reaction time data for each subject.

\section{Results \\ Behavioral}

Repeated measures $2 \times 3$ ANOVAs were conducted on accuracy and RT to assess main effects of the Stroop condition, effects of aversive primes, and interaction between Stroop condition and prime type. A summary of the behavioral results is presented in Table 1. The results indicated that reaction time on correct trials was significantly affected by the content of the Stroop array (congruent, incongruent or stars) $(F(2,12)=53.2, p<.0001)$. Pairwise comparisons indicated that the RT was significantly slower during incongruent trials than congruent trials $(F(1,13)=114.73, p<.0001)$. Reaction times were fastest during the stars condition, and were significantly faster than incongruent $(F(1,13)=51.5, p<.0001)$, but not congruent trials. There were no significant effects of Stroop condition on accuracy $(\mathrm{F}(2,12)=1.27, p=.32)$.

Comparisons between aversive and neutral primes were carried out on the stars trials, in order to assess the effects of the aversive prime without effects of the Stroop task. The behavioral data indicated that during stars trials, the aversive primes had no significant effect on reaction time $(F(1,13)=.75, p=.4)$ or accuracy $(F(1,13)=0.0, p=.97)$ relative to neutral primes.

Consistent with our hypothesis, there was a significant interaction between emotionality and content of the Stroop array on reaction time $(F(2,12)=3.99, p=.047)$. Pairwise comparisons indicated that reaction times on the incongruent trials were slowed further when they were paired with a preceding aversive compared to neutral stimulus $(F(1,13)=6.93, p=.021)$, but no effect of emotion was seen during congruent trials. There were no significant interactions observed between emotionality and the content of the Stroop array on accuracy measures.

\section{Imaging Data}

For the main effect of Stroop array, the voxel-based analyses indicated that significantly greater activation was elicited in DECS regions during incongruent trials relative to congruent trials, 
including the left middle frontal gyrus, posterior parietal cortex, cingulate gyrus and right thalamus (Figure 2). Table 2 lists areas that showed significantly greater activation to incongruent than congruent trials, and those more active to congruent than incongruent trials. Areas more active to aversive than neutral primes included regions within the VAPS, including the right orbitofrontal cortex and posterior cingulate gyrus (Figure 3). Table 3 lists the regions more active to aversive than neutral primes, and those more active to neutral than aversive primes.

We then tested whether brain areas involved in processing of congruent and incongruent trials were differentially affected by the aversive prime relative to the neutral prime. Table 4 lists the regions that showed significant differences between conditions for each contrast. Contrary to our hypothesis, during incongruent trials there were no regions that reached significance for attenuated activity in the presence of the aversive prime during processing of incongruity (Neutral-Incongruent greater than Aversive-Incongruent). During congruent trials, however, a larger network of regions showed emotional attenuation, including the insula, superior frontal gyrus, and superior temporal gyrus (Neutral-Congruent greater than Aversive-Congruent) (Figure 4A, Table 4). Figure 4B illustrates this relationship for percent signal change within all voxels in an anatomically defined region encompassing the inferior frontal gyrus. Areas showing greater activity during aversive than neutral primes for incongruent trials (AversiveIncongruent greater than Neutral-Incongruent) included the inferior frontal gyrus and middle temporal gyrus (Figure 5, Table 4). No areas were found to be significantly enhanced by the aversive primes during congruent trials.

Finally, we tested the relationship between task performance and the effect of the aversive primes on processing of the Stroop array. Voxel-wise correlation analyses were run to examine whether there was a relationship between reaction time and the difference in activity between aversive and neutral primes during incongruent trials. We note that while in many regions the group as a whole did not show a significant mean difference in activity during the AversiveIncongruent minus Neutral-Incongruent contrast, this analysis tested whether individual variability in this difference predicted task performance with no pre-selection for significant voxels. Consistent with our hypothesis, activity in the dIPFC (middle frontal gyrus) was found to show a significant negative relationship with RT on the Aversive-Incongruent minus Neutral-Incongruent contrast, where faster RTs were associated with less emotion-related attenuation of activity. A network of additional regions was also found to show this negative relationship with RT, including the inferior frontal gyrus, insula, middle temporal gyrus, and cingulate gyrus (Figure 6, Table 5). Figure 6B illustrates the significant negative relationship ( $p=.03, R=-.59$ ) between RT and percent signal change during the Aversive-Incongruent minus Neutral-Incongruent contrast, within all voxels in an anatomically defined region encompassing the inferior frontal gyrus and insula. As expected, when examining the opposite contrast (Neutral-Incongruent minus Aversive-Incongruent), we found a similar network of regions showing the opposite positive relationship to RT (Table 5). No areas were found to show a positive correlation with RT where aversive activity was greater than neutral, nor were any found to show a negative correlation where neutral activity was greater than aversive. Furthermore, no regions were found to have a significant relationship with RT during congruent trials (i.e., Neutral-Congruent versus Aversive-Congruent).

\section{Discussion}

This study aimed to answer whether pre-activation of the VAPS through emotional priming would elicit differential DECS engagement during congruent and incongruent trials in a number Stroop task. Furthermore, we examined how these differential effects of the aversive primes would influence executive task performance. As we had hypothesized, we found that performance during incongruent trials was slower during aversive primes compared to 
congruent trials, and that this slowing was related to relative deactivation of the dlPFC during aversive versus neutral trials. However, we did not find that the aversive primes generally led to prefrontal deactivation on incongruent trials, but instead found that congruent trials were associated with emotion-related deactivation across a network of regions including the vlPFC, insula and cingulate gyrus. These results suggest that increased requirements for executive function on incongruent trials may lead to a counteracting effect against the preceding emotionrelated suppression of activity in these areas. Under conditions where the VAPS is primed, these areas contribute to speed of executive performance depending upon their degree of engagement.

The dorsolateral PFC has been identified to be particularly susceptible to attenuated activity when aversive distracters are presented during an executive task. Several studies have found that the dlPFC is particularly involved during sustained working memory and selective attention tasks, and that reductions in its activity due to aversive distracters or simultaneous stressors impairs performance on these executive tasks (Arnsten \& Goldman-Rakic, 1998; Dolcos \& McCarthy, 2006). However, in our emotional priming design, neither the dlPFC nor any other regions were found to show an emotional attenuation effect on incongruent trials. Activation of the VAPS circuitry prior to executive engagement therefore appears to lead to different effects on the DECS circuitry, in that the need for executive processing can override the deactivating effect of aversive stimuli. Although few studies have directly examined the effects of emotional priming on a subsequent executive response, a study by Blair et al. (2007) used a design where emotional stimuli were presented both before and during an executive task (without distinguishing the two effects) and did not find attenuated emotionrelated activity in the dlPFC and anterior cingulate. While it is possible that habituation effects from repetition of photos across the experiment could have contributed to reducing an emotional attenuation effect in the dlPFC, we believe that the patterns seen in the current data likely reflect the dynamic interaction between the emotional and executive systems, where the requirement for executive function during incongruent trials can also counteract the emotional attenuation effect.

The data in the current study also indicated that the vlPFC and insula activity was attenuated by the aversive primes during congruent trials. As the vlPFC in particular is known to be involved in selection processes among competing stimuli (Jha, Fabian, \& Aguirre, 2004), it would be expected that these areas should be engaged under conditions with the most competition (e.g., the aversive prime with incongruent trials). During congruent trials, however, there should be decreased requirements for these selection processes, as there would be less competition between the processes of reading and counting the number of digits. Indeed, the current study indicated that the vlPFC was more active to incongruent than congruent trials, and it showed an additive response in the right hemisphere where activity was greater during the Aversive-Incongruent minus Neutral-Incongruent contrast, but not during the AversiveCongruent minus Neutral-Congruent contrast. It may be therefore that while the aversive primes have a more automatic attenuation effect on the vlPFC and insula during congruent trials, the need for greater selection requirements during incongruent trials counteracts this effect.

In the study by Blair et al. (2007), which used emotional primes simultaneously with emotional interference in the delay period of a Stroop matching task, the authors similarly found that activity in the vIPFC showed differential activity to emotional and neutral interference during congruent trials, while responses to emotional and neutral distracters were similar for perceptual control and incongruent trials. However, their study indicated the opposite pattern for congruent trials, where emotion enhanced, rather than disrupted, activity in the left vlPFC (Brodmann area 47). This difference from the current study's findings provides an illustration of the importance of timing of the VAPS and DECS engagement. Because the study by Blair 
et al. introduced an additional emotional stimulus following the onset of the Stroop task, the vlPFC may have been responsive to the second instance of the emotional interference. Because the current study introduces no additional emotional stimuli following the onset of the Stroop task, it would not be expected to engage in a similar additional response during aversive trials. These differences may reflect the importance of the timing in which emotional and executive systems are engaged in determining how top-down executive effects influence emotional processing.

An alternative view of the current study's findings may be illuminated through the attentional blink phenomenon. In the classic attentional blink task which uses a rapid serial visual presentation of images, attentional processing of an item (T1) impairs the ability to process another item (T2) that follows closely in time (200-500 msec). Several studies have demonstrated that presentation of an emotional stimulus as the T1 item extends the attentional blink, where the arousal induced by the T1 stimulus further decreases processing of the subsequent T2 stimulus (Flaisch, Junghofer, Bradley, Schupp, \& Lang, 2008; Mathewson, Arnell, \& Mansfield, 2008). Similarly, modulation of the attention allocated to T2 (e.g,, by making it emotionally salient or attention-grabbing) has been shown to reduce the attentional blink, where the decrease in processing after a T1 stimulus is overridden by the attention allocated to the salient second stimulus (Shapiro, Caldwell, \& Sorensen, 1997; Trippe, Hewig, Heydel, Hecht, \& Miltner, 2007). Although the very brief presentation of the aversive prime in the current study $(150 \mathrm{msec})$ is likely too short to elicit an attentional blink, it could be that the emotional attenuation seen during congruent trials reflects a similar mechanism where activity across a network of regions is automatically reduced by the aversive primes, and that this phenomenon is modulated by the executive requirements of the subsequent incongruent trials.

The top-down process of regulating responses to task-irrelevant emotional information is known to recruit lateral prefrontal regions (areas 46,9,6/8), orbital cortex (areas 10,11), and medial frontal regions (Beauregard, Levesque, \& Bourgouin, 2001; Blair et al., 2007; Levesque et al., 2003). In the current study we found that activity in these same areas predicted performance on the Stroop task, in that that the attenuation or enhancement of aversive prime processing during incongruent trials was related to speed of executive performance. As the executive override of emotional attenuation during incongruent trials (that is, enhanced rather than reduced activity in response to aversive primes) has a beneficial effect on processing speed in these same regions, it suggests that this process may reflect the engagement of regulatory mechanisms. Blair et al. (2007) have suggested that these regulatory processes occur not only during active inhibition to downplay the effect of emotionally disturbing stimuli, but that they also engage automatically in response to emotional distracters that interfere with executive function. The data in the current study also support this idea of automatic engagement of regulatory mechanisms, and suggest that these mechanisms can not only reduce activity related to the aversive primes, but that they can also enhance emotional processing to the benefit of executive performance speed.

The data overall suggests that emotional priming provides a unique manipulation of the interaction between the DECS and VAPS, where it automatically attenuates activity in the left vlPFC and insula under conditions of low executive requirements. During greater executive requirements, however, activity is enhanced in these regions to counteract this emotion-related attenuation. The data is consistent with Pessoa's model (2008) of functionally integrated cognitive and emotional systems, where executive engagement in a multitude of regions is dependent upon concurrent emotional information being processed. The orchestration between the emotional and executive systems likely depends on the nature of the executive task, the salience and arousal of the emotional stimuli, and the order in which emotional and executive processes are engaged. Future studies examining these factors using electrophysiological 
methods will be able to contribute improved temporal dissociation of neural activity related to emotional primes from subsequent executive processing.

\section{Supplementary Material}

Refer to Web version on PubMed Central for supplementary material.

\section{Acknowledgments}

Support was provided by NICHD T32-HD40127 and NINDS P01-NS41328-01.

\section{References}

Arnsten AF, Goldman-Rakic PS. Noise stress impairs prefrontal cortical cognitive function in monkeys: evidence for a hyperdopaminergic mechanism. Arch Gen Psychiatry 1998;55(4):362-368. [PubMed: 9554432]

Barbas H. Anatomic basis of cognitive-emotional interactions in the primate prefrontal cortex. Neurosci Biobehav Rev 1995;19(3):499-510. [PubMed: 7566750]

Beauregard M, Levesque J, Bourgouin P. Neural correlates of conscious self-regulation of emotion. J Neurosci 2001;21(18):RC165. [PubMed: 11549754]

Beckmann CF, Jenkinson M, Smith SM. General multilevel linear modeling for group analysis in FMRI. Neuroimage 2003;20(2):1052-1063. [PubMed: 14568475]

Blair KS, Smith BW, Mitchell DG, Morton J, Vythilingam M, Pessoa L, et al. Modulation of emotion by cognition and cognition by emotion. Neuroimage 2007;35(1):430-440. [PubMed: 17239620]

Dichter GS, Bellion C, Casp M, Belger A. Impaired Modulation of Attention and Emotion in Schizophrenia. Schizophr Bull. 2008

Dolcos F, McCarthy G. Brain systems mediating cognitive interference by emotional distraction. J Neurosci 2006;26(7):2072-2079. [PubMed: 16481440]

Flaisch T, Junghofer M, Bradley MM, Schupp HT, Lang PJ. Rapid picture processing: affective primes and targets. Psychophysiology 2008;45(1):1-10. [PubMed: 17910736]

Genovese CR, Lazar NA, Nichols T. Thresholding of statistical maps in functional neuroimaging using the false discovery rate. Neuroimage 2002;15(4):870-878. [PubMed: 11906227]

Gray JR, Braver TS, Raichle ME. Integration of emotion and cognition in the lateral prefrontal cortex. Proc Natl Acad Sci U S A 2002;99(6):4115-4120. [PubMed: 11904454]

Hartley LR, Adams RG. Effect of noise on the Stroop Test. J Exp Psychol 1974;102(1):62-66. [PubMed: 4809657]

Jenkinson M, Bannister P, Brady M, Smith S. Improved optimization for the robust and accurate linear registration and motion correction of brain images. Neuroimage 2002;17(2):825-841. [PubMed: 12377157]

Jenkinson M, Smith S. A global optimisation method for robust affine registration of brain images. Med Image Anal 2001;5(2):143-156. [PubMed: 11516708]

Jha AP, Fabian SA, Aguirre GK. The role of prefrontal cortex in resolving distractor interference. Cogn Affect Behav Neurosci 2004;4(4):517-527. [PubMed: 15849894]

Lane RD, Reiman EM, Bradley MM, Lang PJ, Ahern GL, Davidson RJ, et al. Neuroanatomical correlates of pleasant and unpleasant emotion. Neuropsychologia 1997;35(11):1437-1444. [PubMed: 9352521]

Lang, PJ.; Bradley, MM.; Cuthbert, BN. International affective picture system (IAPS): Digitized photographs, instruction manual and affective ratings. Gainesville, FL: The Center for Research in Psychophysiology, University of Florida; 2005. Technical report A-6

Levesque J, Eugene F, Joanette Y, Paquette V, Mensour B, Beaudoin G, et al. Neural circuitry underlying voluntary suppression of sadness. Biol Psychiatry 2003;53(6):502-510. [PubMed: 12644355]

Mathewson KJ, Arnell KM, Mansfield CA. Capturing and holding attention: the impact of emotional words in rapid serial visual presentation. Mem Cognit 2008;36(1):182-200. 
Mitchell DG, Nakic M, Fridberg D, Kamel N, Pine DS, Blair RJ. The impact of processing load on emotion. Neuroimage 2007;34(3):1299-1309. [PubMed: 17161627]

Most SB, Chun MM, Widders DM, Zald DH. Attentional rubbernecking: cognitive control and personality in emotion-induced blindness. Psychon Bull Rev 2005;12(4):654-661. [PubMed: 16447378]

Ochsner KN, Gross JJ. The cognitive control of emotion. Trends Cogn Sci 2005;9(5):242-249. [PubMed: 15866151]

Pansky A, Algom D. Comparative judgment of numerosity and numerical magnitude: attention preempts automaticity. J Exp Psychol Learn Mem Cogn 2002;28(2):259-274. [PubMed: 11911383]

Paradiso S, Johnson DL, Andreasen NC, O'Leary DS, Watkins GL, Ponto LL, et al. Cerebral blood flow changes associated with attribution of emotional valence to pleasant, unpleasant, and neutral visual stimuli in a PET study of normal subjects. Am J Psychiatry 1999;156(10):1618-1629. [PubMed: 10518175]

Pessoa L. On the relationship between emotion and cognition. Nat Rev Neurosci 2008;9(2):148-158. [PubMed: 18209732]

Schupp HT, Stockburger J, Bublatzky F, Junghofer M, Weike AI, Hamm AO. Explicit attention interferes with selective emotion processing in human extrastriate cortex. BMC Neurosci 2007;8:16. [PubMed: 17316444]

Schupp HT, Stockburger J, Codispoti M, Junghofer M, Weike AI, Hamm AO. Selective visual attention to emotion. J Neurosci 2007;27(5):1082-1089. [PubMed: 17267562]

Shapiro KL, Caldwell J, Sorensen RE. Personal names and the attentional blink: a visual "cocktail party" effect. J Exp Psychol Hum Percept Perform 1997;23(2):504-514. [PubMed: 9104007]

Smith SM. Fast robust automated brain extraction. Hum Brain Mapp 2002;17(3):143-155. [PubMed: 12391568]

Smith SM, Jenkinson M, Woolrich MW, Beckmann CF, Behrens TE, Johansen-Berg H, et al. Advances in functional and structural MR image analysis and implementation as FSL. Neuroimage 2004;23 (Suppl 1):S208-219. [PubMed: 15501092]

Trippe RH, Hewig J, Heydel C, Hecht H, Miltner WH. Attentional Blink to emotional and threatening pictures in spider phobics: electrophysiology and behavior. Brain Res 2007;1148:149-160. [PubMed: 17367765]

Voyvodic JT. Real-time fMRI paradigm control, physiology, and behavior combined with near real-time statistical analysis. Neuroimage 1999;10(2):91-106. [PubMed: 10417244]

Vuilleumier P, Richardson MP, Armony JL, Driver J, Dolan RJ. Distant influences of amygdala lesion on visual cortical activation during emotional face processing. Nat Neurosci 2004;7(11):1271-1278. [PubMed: 15494727]

Williams JM, Mathews A, MacLeod C. The emotional Stroop task and psychopathology. Psychol Bull 1996;120(1):3-24. [PubMed: 8711015]

Woolrich MW, Behrens TE, Beckmann CF, Jenkinson M, Smith SM. Multilevel linear modelling for FMRI group analysis using Bayesian inference. Neuroimage 2004;21(4):1732-1747. [PubMed: 15050594]

Woolrich MW, Ripley BD, Brady M, Smith SM. Temporal autocorrelation in univariate linear modeling of FMRI data. Neuroimage 2001;14(6):1370-1386. [PubMed: 11707093]

Young MP, Scannell JW, Burns GA, Blakemore C. Analysis of connectivity: neural systems in the cerebral cortex. Rev Neurosci 1994;5(3):227-250. [PubMed: 7889215] 


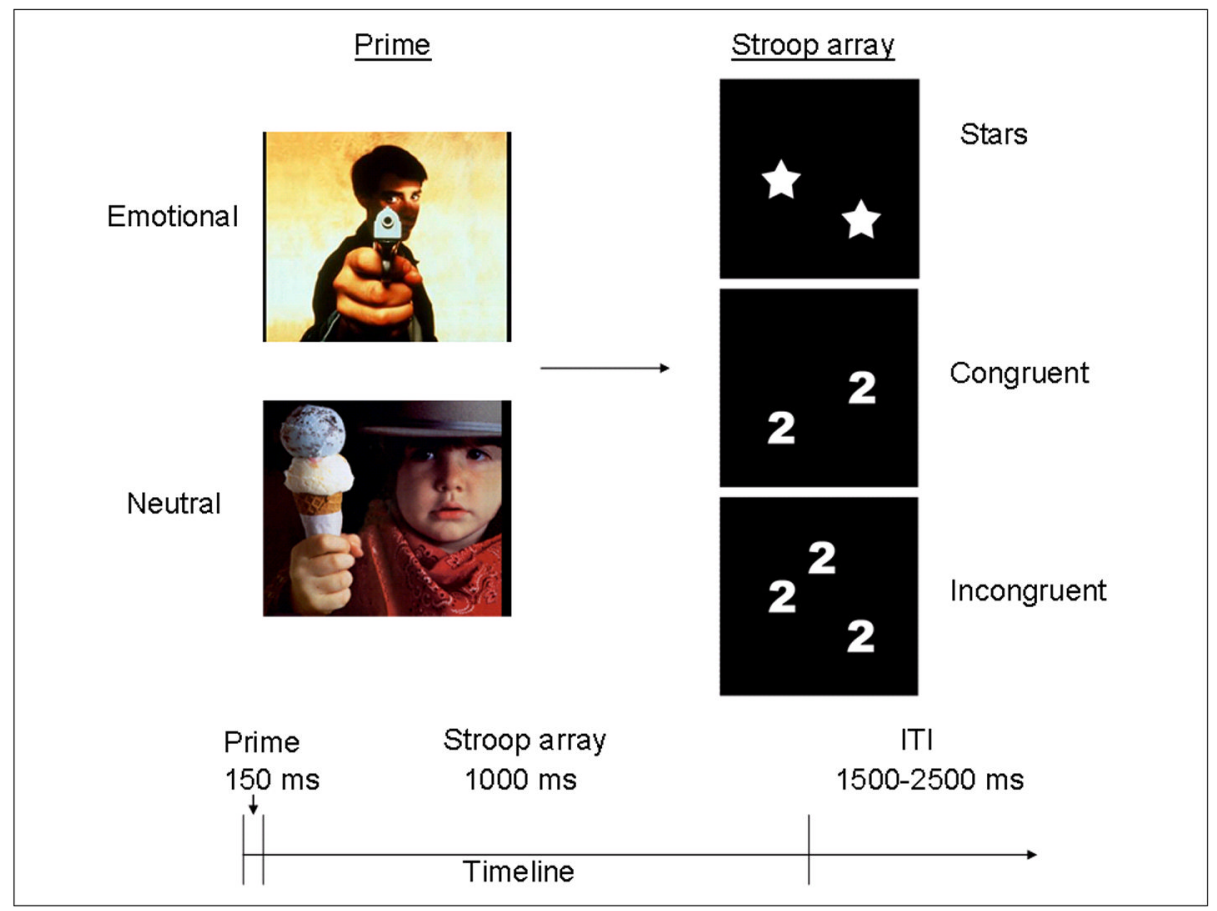

Figure 1.

Schematic of the task design. Each Stroop array (stars, congruent or incongruent) was preceded by an aversive or neutral prime stimulus. ITI = intertrial interval. 


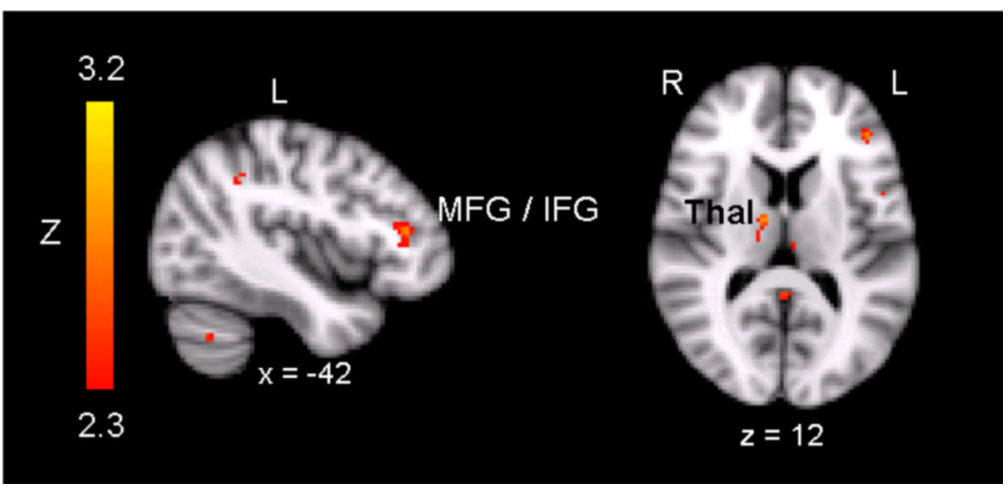

Figure 2.

Main effect of incongruent versus congruent Stroop array during the emotional priming task. $\mathrm{IFG}=$ inferior frontal gyrus; $\mathrm{MFG}=$ middle frontal gyrus; Put = putamen; Thal $=$ thalamus . 


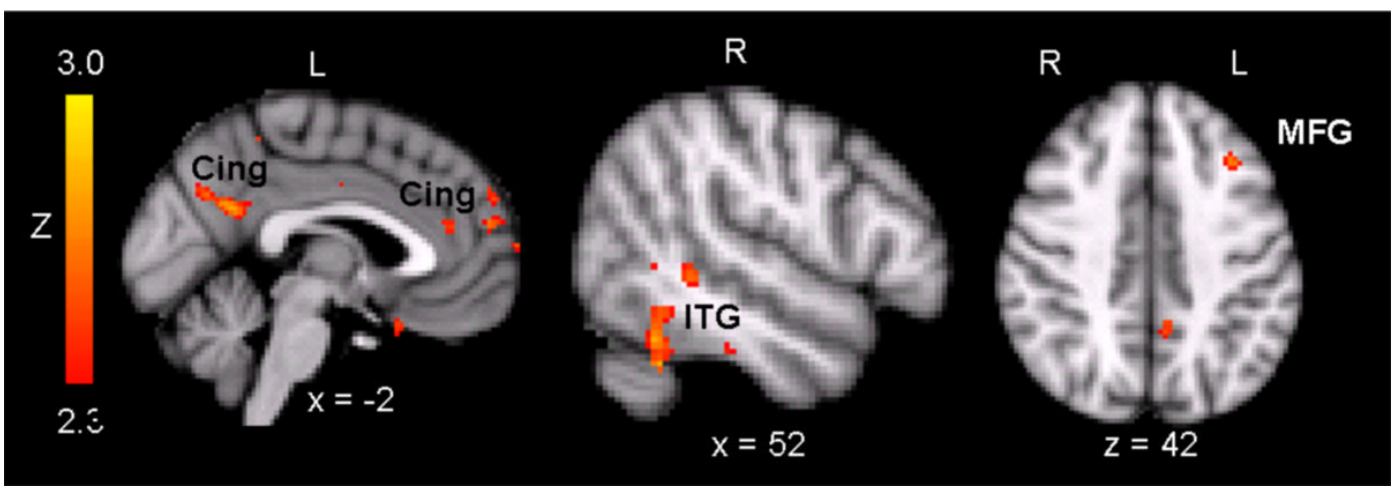

Figure 3.

Main effect of aversive versus neutral prime during Stars trials. Cing = cingulate gyrus; ITG = inferior temporal gyrus; MFG = middle frontal gyrus. 

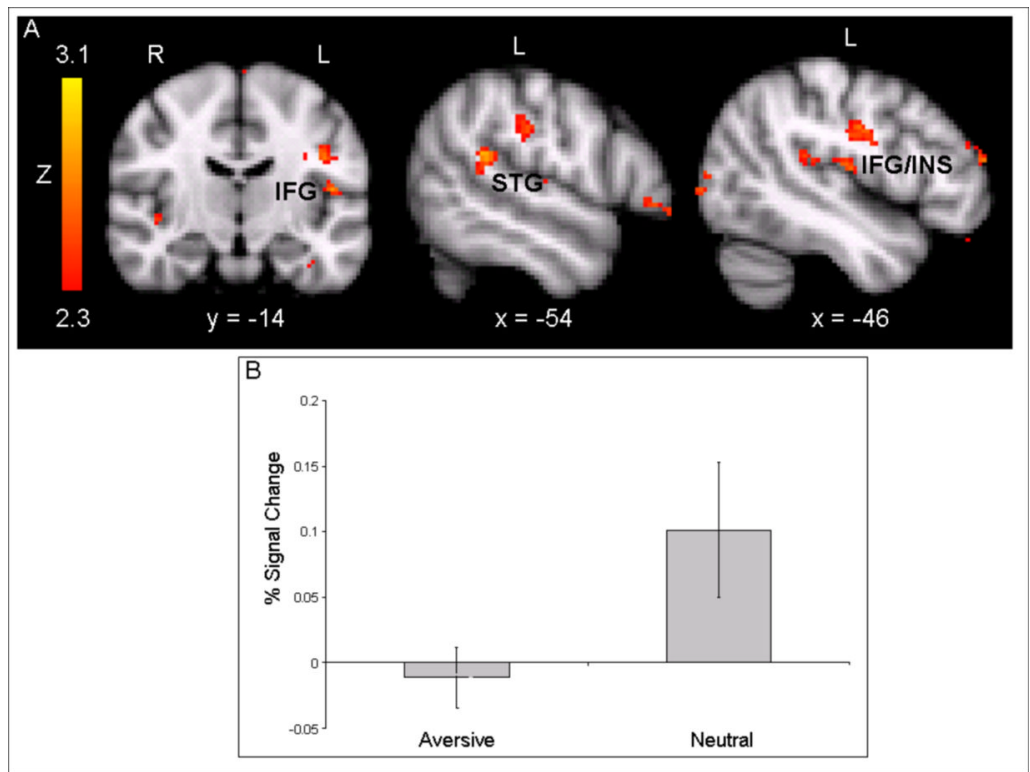

Figure 4.

A) Areas showing significantly greater activation during neutral than aversive primes during congruent trials. IFG $=$ inferior frontal gyrus; INS $=$ insula; $\mathrm{STG}=$ superior temporal gyrus. B) Illustration of percent signal change extracted from the IFG during congruent trials. 


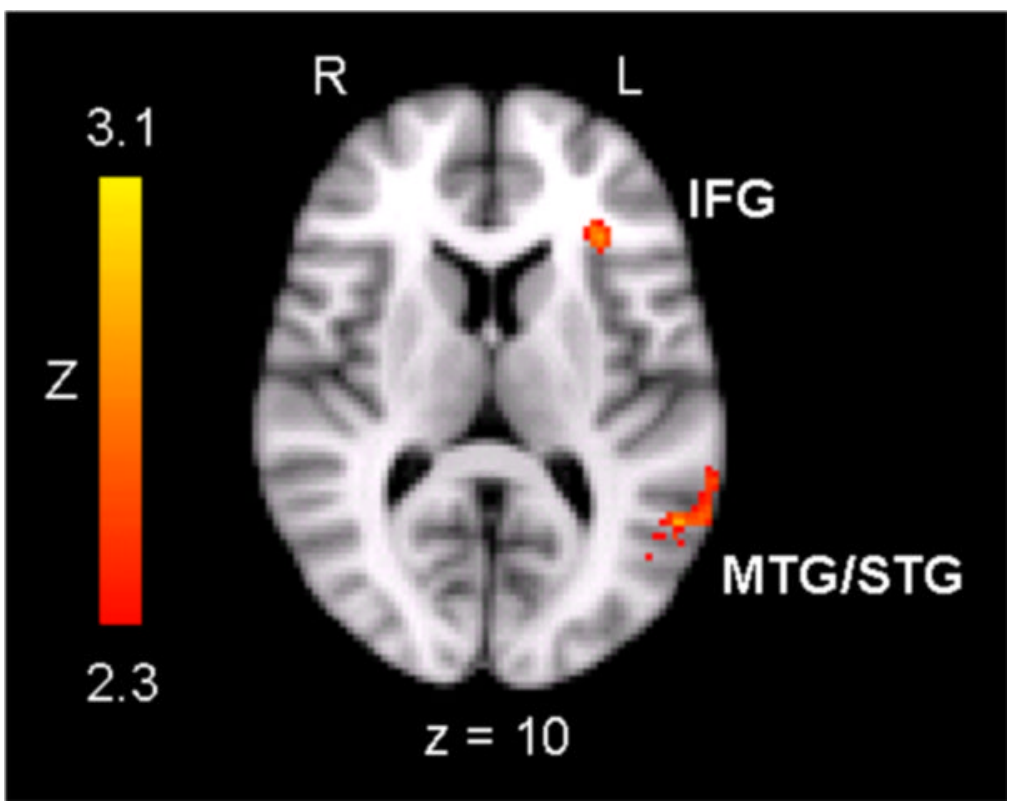

Figure 5.

Areas showing greater activation during aversive than neutral primes during incongruent trials. $\mathrm{IFG}=$ inferior frontal gyrus; $\mathrm{MTG}=$ middle temporal gyrus; $\mathrm{STG}=$ superior temporal gyrus . 

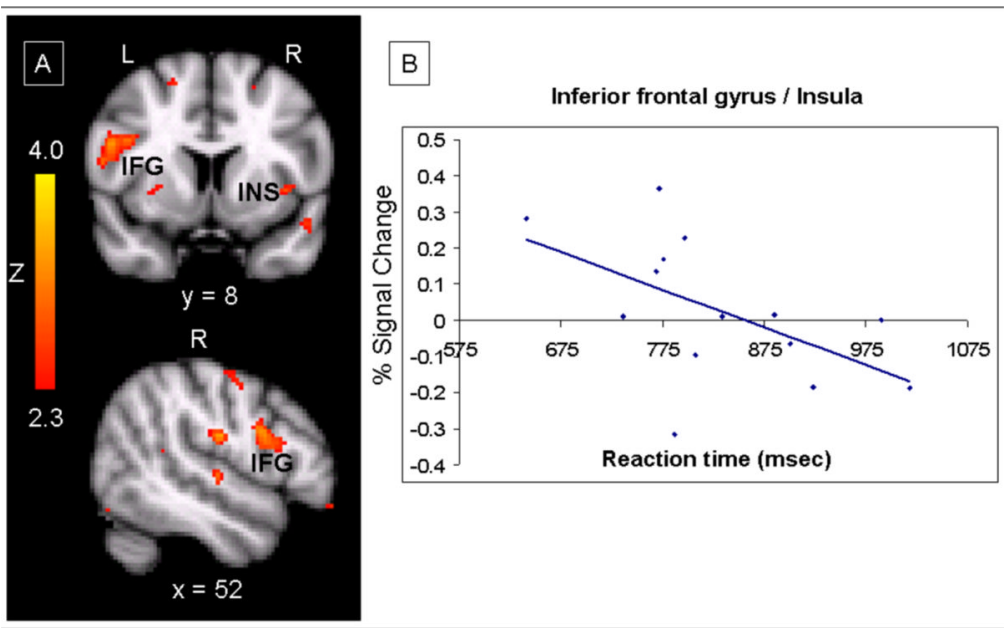

Figure 6.

A) Correlation maps between reaction time and activity in areas that showed significantly greater activation during aversive than neutral primes during incongruent trials. INS = insula; IFG = inferior frontal gyrus. B) Relationship between individual subjects' reaction times during incongruent trials and percent signal change within the IFG and INS during the AversiveIncongruent minus Neutral-Incongruent condition. The correlation with reaction time in the region combining the IFG and INS was significant at $p=.03(\mathrm{R}=-.59)$. 


\section{Table 1}

Behavioral Results

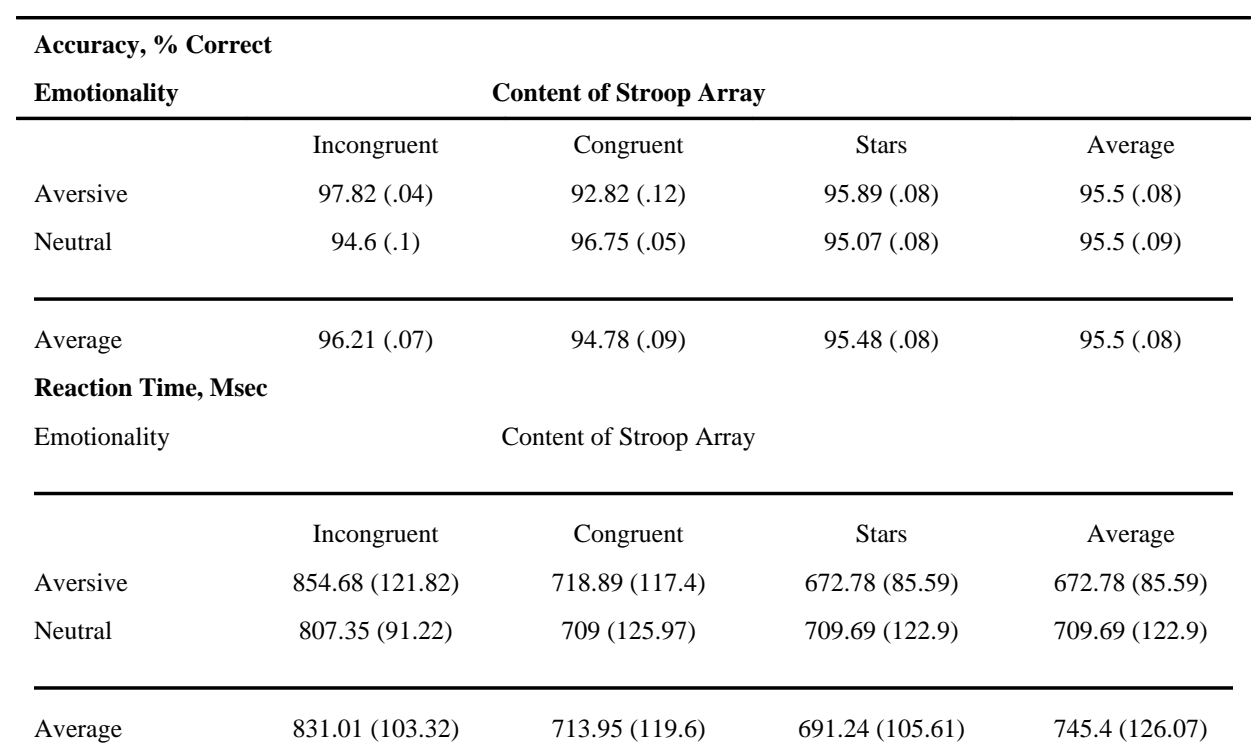




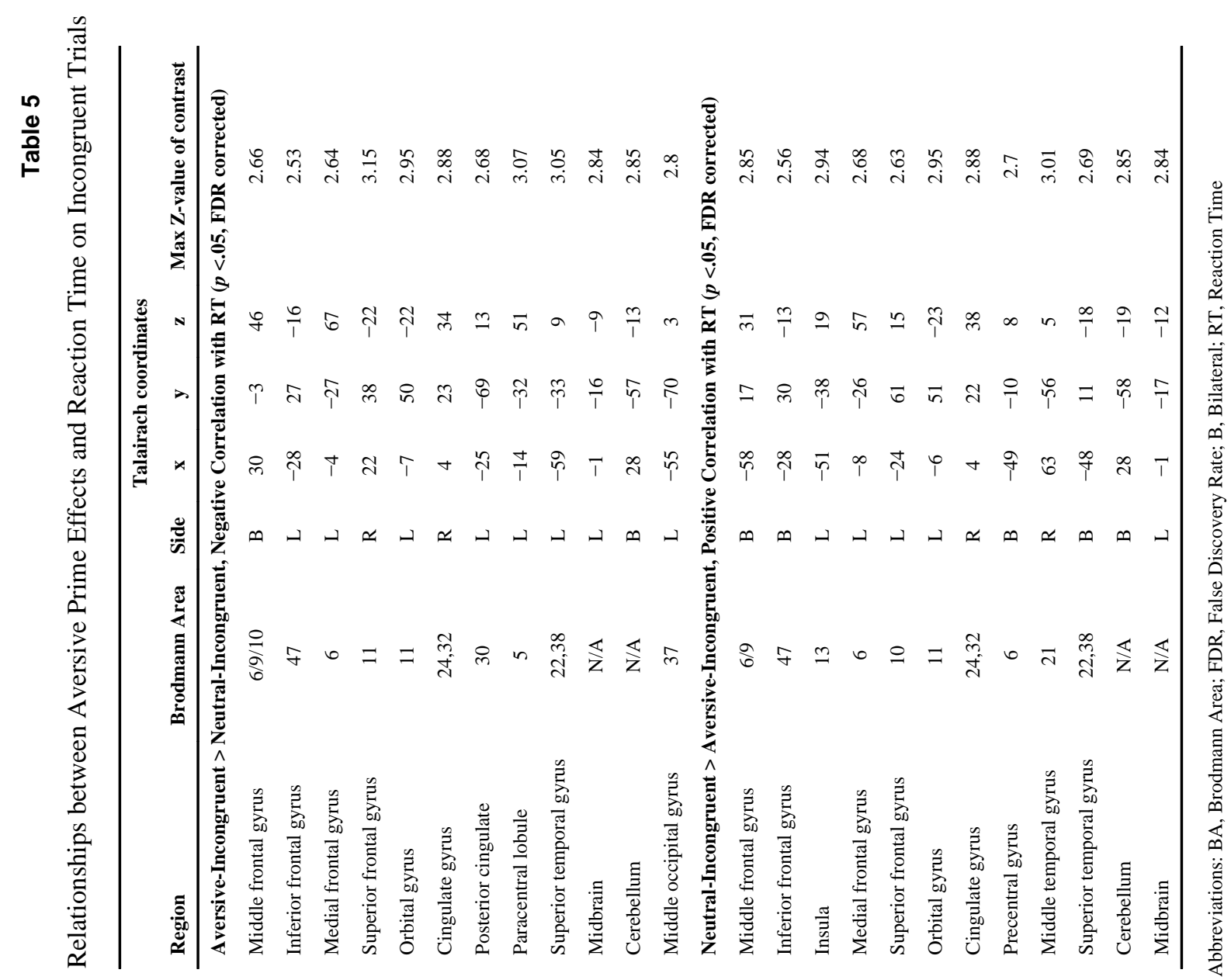

Neuroimage. Author manuscript; available in PMC 2011 February 1. 